\title{
A dynamic machine tool circle test calibration method by R-test
}

\author{
Qian dehou ${ }^{1}$, qingzhen $\mathrm{Bi}^{1}$ \\ ${ }^{1}$ School of Mechanical Engineering, Shanghai Jiao Tong University, Shanghai, 200240, PR China
}

\begin{abstract}
With the continuous popularization of automation in the manufacturing industry and continuous improvement of production efficiency, new requirements have been put forward for the error detection of machine tool. As a new automated detection tool, R-test has been widely studied by scholars. This paper proposes a dynamic test method for R-test, and analyses the influence of three main error sources of servo error, pitch error and reading error. The corresponding elimination methods are also introduced. An experiment on a double turntable machine tool was conducted to verify the analysis. In addition, the selection of feed rate and initial position in circle test is also studied.
\end{abstract}

\section{Introduction}

The precision inspection technology is used to guarantee the stable and high-precision operation of CNC machine tools. Among them, the five-axis machine tool has introduced two rotating axes which induced the nonlinearity of its kinematics model. That is not only a new challenge for the machining process, but also a new subject for error detection of the machine tool.

For detecting the added two rotating shaft of the fiveaxis machine tool, the researchers have proposed many special measuring devices as well as corresponding theoretical models. In the academic, the error test methods popularly studied are mainly divided into two categories, one is to design simple trajectories to directly obtain the specified errors ${ }^{[1,5,6]}$, the other is to measure multiple trajectories and substitute the obtained data into the established kinematic model, then solve each error by an optimization algorithm ${ }^{[7,8,9]}$. No matter which method requires multiple measurements and massive data.

Compare with static test methods, dynamic test methods can obtain a large amount of data efficiently, also avoiding the cumbersome operation. It avoids the influence of data reading error and position-independent error caused by too little obtained data on the static detection $^{[10]}$, as well as prevents the influence of thermal deformation caused by the environment temperature change ${ }^{[11]}$. It can be seen that dynamic test is an efficient and accurate test method, which will become more and more widely used in automated production.

In the face of applying dynamic test methods, we need to compare the impact on measurement results after applying dynamic measurements, and it is beast to establish a model to obtain the deviation between the dynamic test and static test in geometric errors detection. Because in addition to the geometric errors which we usually identified, some other error source, such as thermal error $^{[9]}$, servo error ${ }^{[10]}$ and the mechanical pitch error ${ }^{[11]}$, will also have impacts on the result of the dynamic error detection. We must judge the sensitivity and robustness of dynamic detection method in order to get more accurately machine errors and corresponding compensation.

The first part of the paper analyses the error which introduced by dynamic test and their respective influence. Then introduced the data processing methods to eliminate the impact of new error source due to dynamic test. In the second part of paper, a simple simulation and experiment with a R-test device are conducted to study the influence of dynamic error of test results. The final part of the paper is the conclusion.

\section{Dynamic test error analysis}

The dynamic error of the machine tool is closely related to the feed rate and feed system of the machine tool, the feed rate has the most significant influence on the dynamic error, which consists of the hysteresis error, reverse jump error, the servo mismatch error and own servo error (contour error).

\subsection{Servo error}

The typical simplified feed control system on an industrial machine tool, can be represented by figure 1 . By the way, the machine tool also employed in the experimental verification.

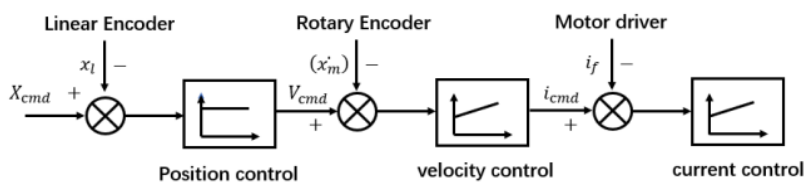

Figure.1 machine tool feed control system 
As show in Fig.1, the feed mechanical system is powered by a rotary servomotor via gearbox and ballscrew. The feed control system consists of three loops: current loop, speed loop and position loop. The servo motor itself provides current feedback, at the same time, the rotary encoder mounted on the servomotor provides feedback on the velocity control loop. The feedback on the position loop is provided by the linear encoder on the rail. In this paper, we mainly discuss the circle test which commonly used in the geometric accuracy measurement. A typical circle test diagram by R-test is presented in figure 2, mounted on the spindle end is the R-test device, which consists of three high-precision displacement sensors. The measurement directions of three sensors intersect at one point and the point is the standard ball center. The circle test is that a series of circular trajectories are taken in the space by rotation axis linked with linear axis and more information can be found in ISO230-4[12]. When using the R-test for the circle test, the machine opens RTCP to ensure the position of the tool tip is unchanged under five-axis linkage movements.

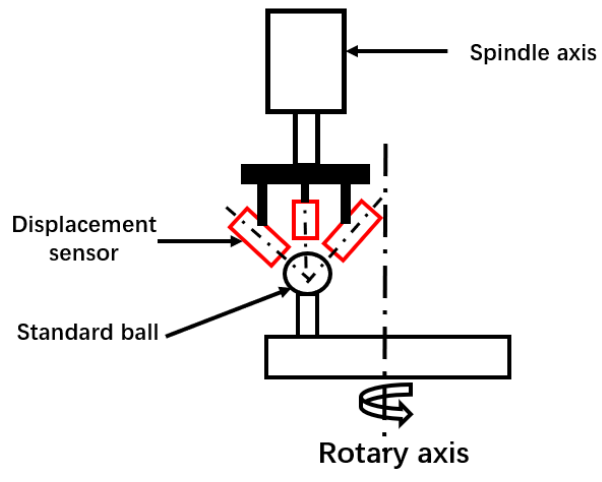

Figure.2 The diagram of R-test

In the circle test, the motion of the linear axis and the rotary axis are gentle, as well as the current loop respond much faster than the velocity loop, so its transfer function could be idealized as unity[13]. Figure 3 shows the structure of three closed control loops of the feed system of a single axis. The feed system controls the servo motor through the current loop, and then controls the speed of axis movement by the encoder on the motor, while the outermost control loop is the position loop of the machine grating-rule sensor.

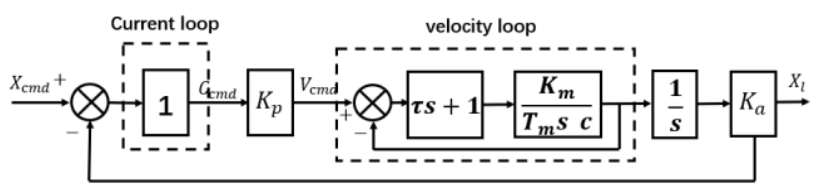

Figure 3. Diagram of the feed control model

Hence, the friction-driven hysteresis model can be used as the mechanical structure of feed drive system. From the diagram shown in Figure 3, the transfer function of velocity loop is given by:

$$
H_{v}(\mathrm{~s})=\frac{w_{n}^{2}(\tau s+1)}{s^{2}+2 \xi w_{n} s+w_{n}^{2}}
$$

Where the $w_{n}$ is the natural frequency, and $\xi$ is the damping ratio. The zero point of the system brings certain oscillation to the velocity control of the machine tool, which also has a certain impact on the dynamic test. If the rotation axis has an eccentricity $\Delta \mathrm{L}$, the input of the circle detection by R-test on the linear axis can be considered as a simple harmonic function:

$$
\left\{\begin{array}{l}
v_{x} \approx \Delta \mathrm{x}=\Delta \mathrm{L} \cos (\mathrm{wt}) \\
v_{y} \approx \Delta y=\Delta L \sin (w t)
\end{array}\right.
$$

Where $v_{x}$ and $v_{y}$ are the velocity of $\mathrm{X}$ axis and $\mathrm{Y}$ axis respectively. $\mathrm{W}$ is the angular velocity of the circular motion, related to the feed rate and the radius of the circle.

Figure 4 is a simulation of the feed system servo nature for circle test, the eccentricity $\Delta \mathrm{L}=0.01 \mathrm{~mm}$, and a circular motion duration $\mathrm{T}=8 \mathrm{~s}$. As shown in Figure 4 (a), a hysteresis can be seen at the beginning, and then the response curve intersected the original curve. The servo response of the machine tool presents an underdamped oscillation state in the circle test. In the figure 4 (b), the dynamic error fluctuates up and down at zero value, which makes the measurement result of the geometric larger than the static test
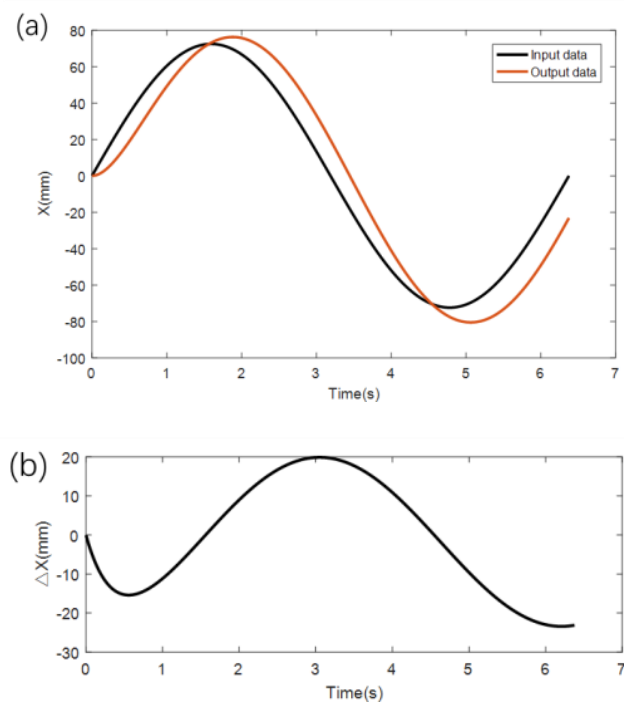

Figure.4 (a)Second-order damping system simulation ; (b) error between the input and output signal

\subsection{Pitch error}

Pitch error is caused by mechanical transmissions such as worm gears and rolling screws, so this error usually has a certain periodicity. Noriyuki Kato[14] simulated and experimentally studied the pitch error in the circle test by using the ballbar. Here, a fast Fourier transform (FFT) method was used to analyse the pitch error, the pitch error was modelled as follows:

$$
E_{p}=\sum_{i}^{n} W_{i} \sin \left(N_{i} R_{a l l} w_{m} t+\varphi_{i}\right)
$$

Where, $R_{\text {all }}$ is the reduction ratio of the mechanical transmission, $W_{i}$ is the amplitude of ith pitch error, $N_{i}$ is the number of pitch error harmonics, and $\varphi_{i}$ is the initial phase of ith pitch error. Pitch error has directional 
dependence, and can be measured in the tangential direction of circle test.

Figure 5 is the amplitude spectrum of a circle test. In the test, the $\mathrm{C}$ axis is rotated, and simultaneously the $\mathrm{X} / \mathrm{Y}$ axis is linked with the $\mathrm{C}$ axis. The stroke is one $\mathrm{CW}$ revolution of the rotary axis, with the angular velocity 0.46 $\mathrm{rad} / \mathrm{s}$. As shown in Figure 5, several peaks can be observed, and the corresponding frequencies of peaks have an integer multiple relationship. The frequency corresponds to the number of teeth of the mechanical transmission structure such as worm gear. The maximum amplitude is $2.2 \times$ $10^{-4} \mathrm{~mm}$, which can be seen that pitch error has little impact on the results of dynamic circle test.

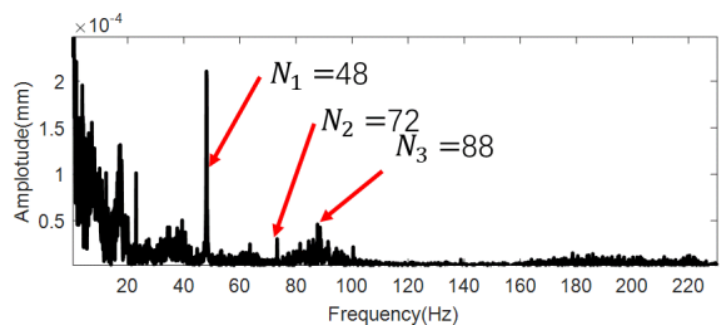

Figure 5. Amplitude spectrum of pitch errors in a circle test

\subsection{Read error}

As with static measurements, continuous measurements by dynamic test can also cause reading errors in the sensor itself. In order to avoid the error during sensor reading for a quasi-static measurement, there are certain requirements for the number of points selected in the ISO230 standard.

Figure 6 shows the results of such a quasi-static measurement performed on a five-axis double turn-table machine tool. The $\mathrm{C}$-axis rotated in $\mathrm{CW}$ direction one revolution while $\mathrm{X} / \mathrm{Y}$ axis linked. 12 and 72 target position uniformly distributed over the circumference are respectively selected for analysis. Each time the selected position reached, the machine tool stops and sensor data is read. Increasing number of targets not only makes the measurement results smoother, but also closer to the actual situation of the machine motion. If the least squares method which was commonly used in error recognition is applied to determine the rotary axis geometric error, fewer selected points may cause the fit circle smaller, resulting in inaccurate recognition.

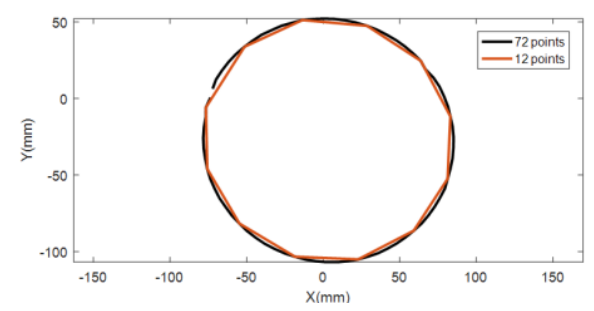

Figure 6. Circle test of different numbers selection

Every data point in the static measurement is used equally, but a large amount of continuous data is obtained in the dynamic measurement. Some signal processing

method can be used to get more rigorous and robust results. Among them, Kalman filtering is the most popular signal processing method in the field of spatial position detection, and it has the following form:

$$
\begin{gathered}
\hat{x}_{k}=F_{k} \hat{x}_{k-1}+B_{k} \overrightarrow{u_{k}} \\
P_{k}=F_{k} P_{k-1} F_{k}^{T}+Q_{k} \\
\hat{x}_{k}^{\prime}=\hat{x}_{k}+K^{\prime}\left(\overrightarrow{z_{k}}-H_{k} \hat{x}_{k}\right) \\
P_{k}^{\prime}=P_{k}-K^{\prime} H_{k} P_{k} \\
K^{\prime}=P_{k} H_{k}^{T}\left(H_{k} P_{k} H_{k}^{T}+R_{k}\right)^{-1}
\end{gathered}
$$

Vector $\mathrm{x}$ is the position parameter to be detected. In a circle test, $\hat{x}=\left[\begin{array}{l}x \\ y \\ W\end{array}\right], \mathrm{x}$ and $\mathrm{y}$ are the position coordinates, $\mathrm{W}$ is the angular velocity of the circle test, related to the machine tool feed and the radius of the circle path, $\mathrm{F}$ is the corresponding state transformation matrix. $K^{\prime}$ is the Kalman gain, which is the core of the Kalman filter. It is obtained from the error distribution of the actual state space and the distribution of measuring error, both errors must satisfy the Gaussian distribution. In the machine tool error detection, the Gaussian parameter of the error distribution can be obtained by the air mining before the machine tool movement. In summary, in the field of data acquisition, dynamic test can obtain better data than static test. In the figure 6, a Kalman filter was applied to deal with the dynamic test data, it can be seen that the deviation of the sensor reading is obviously eliminated, and the curve becomes smooth, mainly depending on the influence of

mechanical

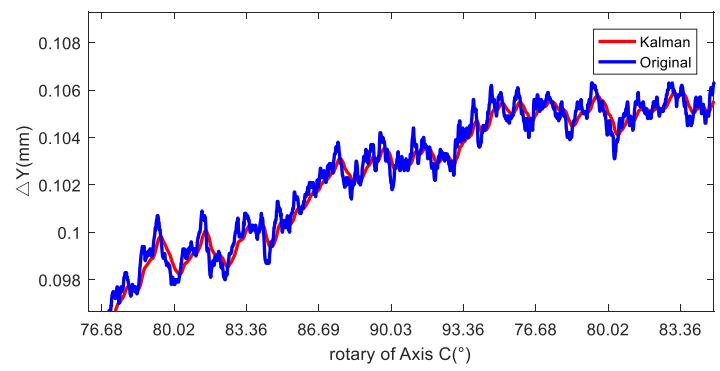

Figure 7. Deal with read error by a Kalman filter

\section{EXPERIMENT}

The experiments were carried out on an industrial five-axis double turntable machine tool. Only $\mathrm{X}$-axis, Yaxis and $\mathrm{C}$-axis were used for test and its structure can be seen in figure 7 . The R-test is mounted on the machine spindle with the standard ball mounted on the rotary table $\mathrm{C}$ as shown in figure 7 . The R-test can perform continuous measurement with a maximum duration of $120 \mathrm{~s}$, sampling frequency is $2000 \mathrm{~Hz}$, and the data is transmitted via WiFi[15]. 


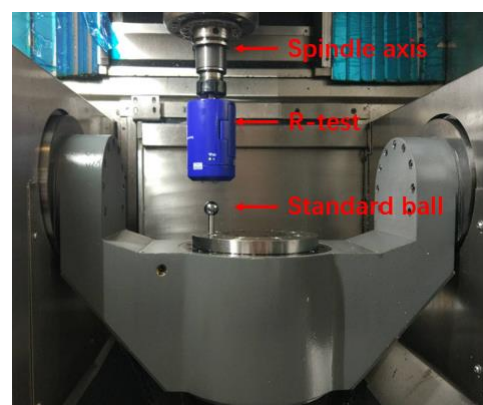

Figure 8. Experimental device structure

In circle test, a clockwise trajectory circle of radius $72.411 \mathrm{~mm}$ was conducted with different feed rate (from 500 to 7000 ). For comparison, the same static test was also done, 72 points of uniform distribution in circle were selected for error detection. Each experiment was repeated three times to ensure the accuracy of the data. The time required for each static measurement is $15 \mathrm{~min}$ while the dynamic measurement is $1 \mathrm{~min}$.

Figure 8 is the result of the experiment. It can be seen that as the feed rate increases, the deviation between the dynamic test and static test becomes larger. When the feed rate reaches 7000 , the standard deviation is $21 \mathrm{um}$ in the $\mathrm{X}$ direction and $15 \mathrm{um}$ in the $\mathrm{Y}$ direction, which cannot be used as data for geometric error identification. This is mainly because the circle test trajectory has an initial velocity at the beginning, there is obvious hysteresis in the initial stage of the motion. Then, under the impact of the servo driver system, the motion is overshot when velocity in the $\mathrm{X}$ direction is close to 0 position. After it passes through the quadrant, it will continue to oscillate and eventually reach the steady state. The $\mathrm{Y}$-axis has the same phenomenon as the $\mathrm{X}$-axis but the hysteresis effect at the initial position is more serious. This is because the circle test starts from $-90^{\circ}$ and the velocity component in the Ydirection is much larger than the $\mathrm{X}$-direction. However, when the feed speed is reduced to 2000, the standard deviation between dynamic test and static test is $4.8 \mathrm{um}$ in the $\mathrm{X}$-direction, and $3.7 \mathrm{um}$ in the $\mathrm{Y}$ direction, which can basically meet the needs of geometric identification. If we fit the feed servo system with a transfer function with one zero and two poles, the fit can reach $98.27 \%$, the result can be seen in the figure 9 .

(a)

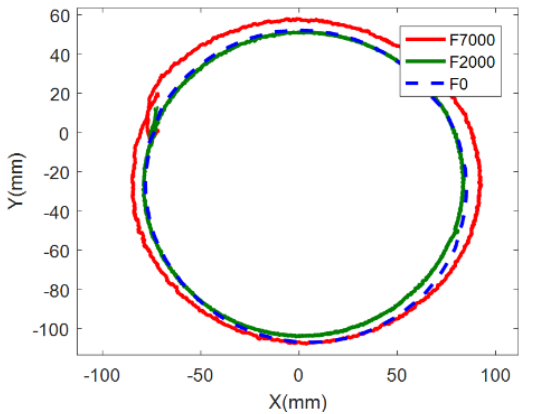

(b)

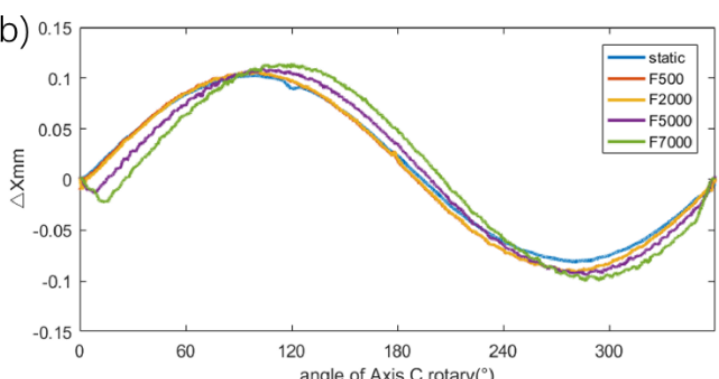

(c)

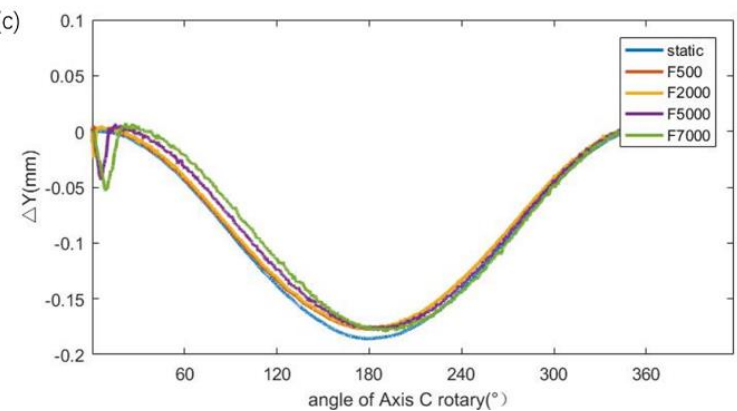

Figure 9. Cicle tests with different feed rates:(a) circle trajectory (b) X-axis (c) Y-axis
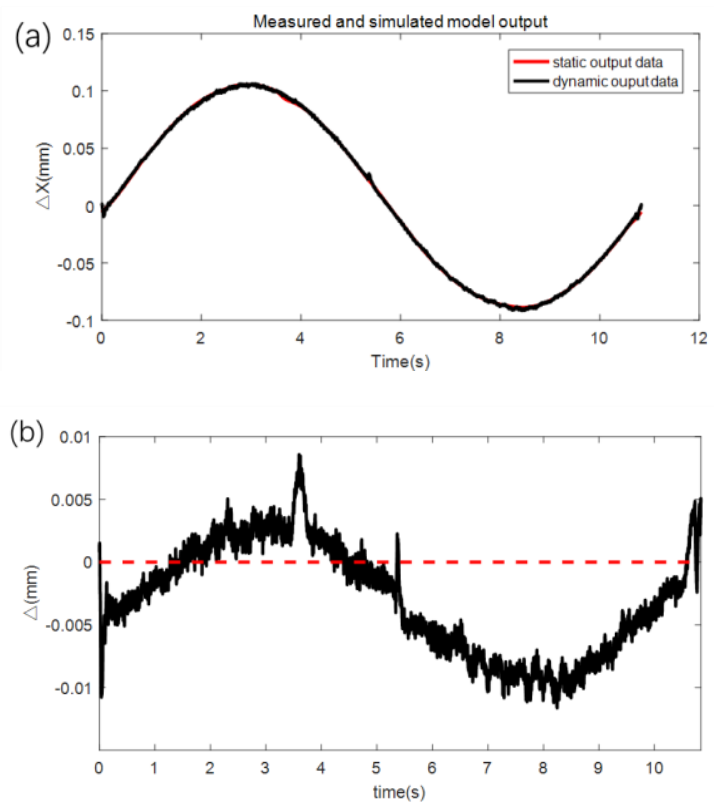

Figure 10. Servo feed system simulation in a dynamic circle test: (a) the $\mathrm{X}$-axis error simulation carve and experiment data carve (b) the difference between simulation and experiment.

In order to obtain closer data to the machine geometric error, we perform some basic processing on the dynamic test data. First the servo driver system is fitted to the second-order damping system, and then a Kalman lowpass filtering is performed to eliminate the influence of the sensor reading error and pitch error. The final test results can be much more accurate.

\section{CONCLUSION}

1) An efficient dynamic test method by using R-test is proposed and compared with static test method. 
2) The performance of the three main error sources of servo error, pitch error and reading error are analysed in dynamic test, as well as corresponding elimination methods are introduced. A data processing flow for obtaining reliable geometric error data from dynamic test is also introduced.

3) Analyze and experiment the influence of the feed rate and the initial position of the circle test on dynamic error measurement.

\section{ACKNOWLEDGEMENTS}

The work is supported by the National Natural Science Foundation of China (Grant No. 51475302 and U1537209) and the Science \& Technology Commission of Shanghai Municipality (Grant No. 17XD1422500), which are highly appreciated by the authors.

\section{References}

1. Jiang X, Cripps R J. A method of testing position independent geometric errors in rotary axes of a fiveaxis machine tool using a double ball bar[J]. International Journal of Machine Tools \& Manufacture, 2015, 89:151-158.

2. Uddin M S, Ibaraki S, Matsubara A, et al. Prediction and compensation of machining geometric errors of five-axis machining centers with kinematic errors[J]. Precision Engineering, 2009, 33(2):194-201.

3. He Z, Fu J, Zhang L, et al. A new error measurement method to identify all six error parameters of a rotational axis of a machine tool[J]. International Journal of Machine Tools \& Manufacture, 2015, 88:1 8.

4. Xiang S, Altintas Y. Modeling and compensation of volumetric errors for five-axis machine tools[J]. International Journal of Machine Tools \& Manufacture, 2016, 101:65-78.

5. Ibaraki S, Oyama C, Otsubo H. Construction of an error map of rotary axes on a five-axis machining center by static R-test[J]. International Journal of Machine Tools \& Manufacture, 2011, 51(3):190-200.
6. Zhu S, Ding G, Qin S, et al. Integrated geometric error modeling, identification and compensation of $\mathrm{CNC}$ machine tools $[\mathrm{J}]$. International Journal of Machine Tools \& Manufacture, 2012, 52(1):24-29.

7. Bringmann B, Knapp W. Machine tool calibration: Geometric test uncertainty depends on machine tool performance[J]. Precision Engineering, 2009, 33(4):524-529.

8. Los A, Mayer J R R. Application of the adaptive Monte Carlo method in a five-axis machine tool calibration uncertainty estimation including the thermal behavior $\vec{s}[\mathrm{~J}]$. Precision Engineering, 2018.

9. Bitar-Nehme E, Mayer J R R. Thermal volumetric effects under axes cycling using an invar R-test device and reference length[J]. International Journal of Machine Tools \& Manufacture, 2016, 105:14-22.

10. Ding W, Zhu X, Huang X. Effect of servo and geometric errors of tilting-rotary tables on volumetric errors in five-axis machine tools $[\mathrm{J}]$. International Journal of Machine Tools \& Manufacture, 2016, 104:37-44.

11. Miller J E, Longstaff A P, Parkinson S, et al. Improved Machine Tool Linear Axis Calibration Through Continuous Motion Data Capture[J]. Precision Engineering, 2016, 47:249-260.

12. ISO 230-4. Test code for machine tools. Circular tests for numerically controlled machine tools. BSI Standards Publication; 2005.

13. Shi S, Jing L, Wang X, et al. Analysis of the transient backlash error in CNC machine tools with closed loops[J]. International Journal of Machine Tools \& Manufacture, 2015, 93(page: 49):49-60.

14. Kato N, Sato R, Tsutsumi M. Analysis of circular trajectory equivalent to cone-frustum milling in fiveaxis machining centers using motion simulator[J]. International Journal of Machine Tools \& Manufacture, 2013, 64(4):1-11.

15. Florussen G H J, Spaan H A M. Dynamic R-Test for Rotary Tables on 5-Axes Machine Tools[J]. Procedia Cirp, 2012, 1:536-539. 